\title{
Arginine and Asymmetric Dimethylarginine in Puromycin Aminonucleoside-Induced Chronic Kidney Disease in the Rat
}

\author{
Gin-Fu Chen $^{a}$ Natasha C. Moningka ${ }^{a}$ Jennifer M. Sasser ${ }^{a}$ Sergey Zharikov ${ }^{b}$ \\ Mark Cunningham, Jr. ${ }^{a}$ You-Lin Tain ${ }^{c}$ Idit F. Schwartz ${ }^{d}$ Chris Baylis ${ }^{a, b}$ \\ Departments of a Physiology and Functional Genomics and ${ }^{\mathrm{b}}$ Medicine, University of Florida, Gainesville, Fla., USA; \\ 'Departments of Pediatrics, Kaohsiung Chang Gung Memorial Hospital, and Chang Gung University College of \\ Medicine, Taiwan, ROC; ${ }^{\mathrm{d}}$ Department of Nephrology, Tel Aviv Sourasky Medical Center, Tel Aviv, Israel
}

\section{Key Words}

Argininosuccinate synthase $\cdot$ Argininosuccinate lyase $\cdot$

Arginase $\cdot$ Cationic amino acid transporter, CAT1 .

Dimethylarginine dimethylaminohydrolase, DDAH •

Hypertension • Proteinuria • Creatinine clearance •

Nitric oxide

\begin{abstract}
Background/Aims: Reduced renal L-arginine (L-Arg) synthesis/transport, induction of arginases and increased endogenous NOS inhibitor, asymmetric dimethylarginine (ADMA) will inhibit NO production. This study investigated pathways of L-Arg synthesis/uptake/utilization, ADMA degradation and oxidant/antioxidants in puromycin aminonucleoside (PAN) chronic kidney disease (CKD). Methods: Rats were given low- (LD) or high-dose (HD) PAN and followed for 11 weeks for proteinuria. BP was measured and blood and tissues were harvested and analyzed for abundance of argininosuccinate synthase (ASS) and lyase (ASL), arginase, cationic amino acid transporter (CAT1) and dimethylargininedimethylaminohydrolase (DDAH) in kidney, cortex, aorta and liver. Arginase and DDAH activity, plasma L-Arg and ADMA, renal pathology and creatinine clearances were also mea-
\end{abstract}

sured. Results: PAN caused dose-dependent kidney damage and hypertension and creatinine clearance fell in HD-PAN. Renal ASS fell in HD-PAN, renal cortex and aortic ASL and membrane CAT1 fell in both PAN groups. There was no activation of renal arginase, but aortic arginase increased in LD-PAN. Renal DDAH activity fell moderately in LD-PAN and markedly in HD-PAN where hepatic DDAH activity also fell. Plasma L-Arg was unchanged while ADMA rose moderately and dose-dependently with PAN. There were several indices of oxidative stress which was most prominent in HD-PAN. Conclusion: Reduction in renal ASS/ASL and loss of renal cortex CAT1 compromises renal L-Arg synthesis and release. Loss of aortic CAT1 impairs L-Arg uptake. Increased plasma ADMA was associated with progressive loss of renal DDAH activity. However, loss of renal clearance and falls in hepatic DDAH activity in HD-PAN did not have additive effects on plasma ADMA.

Copyright $\odot 2011$ S. Karger AG, Basel

Gin-Fu Chen and Natasha C. Moningka contributed equally to this study.

\section{KARGER}

Fax +41613061234 E-Mail karger@karger.ch www.karger.com

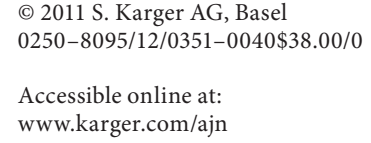

Prof. Chris Baylis, $\mathrm{PhD}$

J. Robert and Mary Cade Foundation

University of Florida

Gainesville, FL 32610-0274 (USA)

E-Mail baylisc@ufl.edu 


\section{Introduction}

Nitric oxide (NO) deficiency develops in patients and animals with chronic and end-stage kidney disease (CKD, ESKD), and contributes to progression and cardiovascular risk [1]. There are multiple potential causes of NO deficiency in $\mathrm{CKD}$, including reduction in the $\mathrm{NO}$ synthase (NOS) substrate, L-arginine (L-Arg) [1] since the normal kidney synthesizes most of the circulating L-Arg; this is by enzymatic conversion of citrulline by argininosuccinate synthase (ASS) and lyase (ASL) [2]. We recently reported impaired renal release of L-Arg in renal mass reduction (RMR)-induced CKD [3]. L-Arg is made in proximal tubules and secreted into plasma via transporters including the cationic amino acid transporter (CAT)1 [4]. CAT1 is also constitutively present on endothelial cells and is required to transport plasma L-Arg into the endothelium [5]. Endothelial L-Arg transport is impaired in renal disease, due both to the presence of circulating inhibitors in patients with ESKD, and to reduction in vascular CAT abundance/activity in rats with RMR-induced CKD $[1,6]$.

The availability of L-Arg for NO synthesis is also determined by the activity of alternate pathways of L-Arg metabolism including arginases [2]. Vascular arginases are elevated in several CKD and hypertension models and may divert L-Arg away from NOS, leading to decreased NO production [1].

Another cause of NO deficiency is accumulation of the endogenous NOS inhibitor asymmetric dimethylarginine (ADMA). ADMA is made during protein methylation by protein arginine methyltransferase (PRMT)1 and is released following proteolysis. Some ADMA is removed by urinary excretion, but the majority is by enzymatic degradation by dimethylarginine-dimethylaminohydrolase (DDAH). The kidney is a major site of ADMA degradation, and renal DDAH activity plays an important role in control of circulating ADMA levels [7]. This may be the main reason why plasma ADMA increases in CKD and ESKD [8]. Increases in plasma ADMA have also been reported in some animal models of CKD including the puromycin aminonucleoside (PAN)-induced model of CKD $[1,9]$.

The goal of this study was to investigate determinants of L-Arg and ADMA metabolism in rats with PAN-CKD. We created moderate and severe models and investigated renal ASS/ASL abundance (indicative of renal L-Arg synthetic capacity), CAT1 abundance in kidney cortex (a measure of proximal tubule L-Arg efflux capacity) and aortic CAT1 abundance (a measure of endothelial L-Arg uptake). We also determined arginase abundance and activity (as a possible competing pathway for L-Arg utilization), plasma ADMA and renal and hepatic DDAH abundance and activity. We further investigated the level of oxidative stress since DDAH abundance and activity are suppressed in the presence of oxidants [7].

\section{Methods}

Male Sprague-Dawley rats $(n=24)$ were used in accordance with NIH guidelines and approved and monitored by the University of Florida IACUC. Rats were in 3 groups ( $n=8 /$ group): control (saline), low-dose (LD-PAN) (25 mg/kg BW initial, $10 \mathrm{mg} / \mathrm{kg}$ boosters), and high-dose (HD-PAN) (50 mg/kg BW initial, $20 \mathrm{mg} /$ $\mathrm{kg}$ BW boosters). PAN was given subcutaneously at week 0 with boosters at weeks 2, 4 and 6. Urine was collected overnight (16 h) in metabolic cages and analyzed for urine protein excretion (UpV) by the Bradford method (BioRad, Hercules, Calif., USA) at weeks $0,1,3,5,7,9$ and 11. At week 11, rats were placed on a low-nitrate diet (ICN AIN 76C, MP Biomedical, Solon, Ohio, USA) prior to overnight urine collection. Rats were anesthetized with isoflurane (Abbott Laboratories), mean blood pressure (BP) was measured via the abdominal aorta, blood was collected, and plasma was stored at $-80^{\circ} \mathrm{C}$. The organs were perfused with PBS, the left kidney removed, weighed and fixed for histology, and aorta, liver and right kidney cortex were flash frozen and stored at $-80^{\circ} \mathrm{C}$.

Western blot analysis for ASS and ASL in kidney cortex was described previously [3], as were DDAH isoforms, SOD isoforms and p22phox Western blot techniques [10]. For arginase, rabbit polyclonal arginase II antibody (Santa Cruz, 1:3,000) with goat anti-rabbit IgG-HRP secondary (Bio-Rad, 1:3,000). Chicken arginase I antibody (gift from Dr. Sidney Morris) was used at 1:10,000 with goat anti-chicken IgG-HRP secondary antibody. For CAT1, kidney cortex and aorta were fractionated into membrane and cytosolic fractions and probed as described [6] and nitrotyrosine was run on a $7.5 \%$ gel $(50 \mathrm{~mA}, 21 / 2 \mathrm{~h})$ with primary antibody (Upstate, $05-233$ ) at 1:500 and goat anti-mouse secondary (BioRad 170-6516) at 1:2,000. All membranes were corrected for total protein loaded (from Ponceau Red stain; Sigma, St. Louis, Mo., USA) and appropriate positive control so that protein abundance was represented as IOD/Ponceau/control.

Arginase activity was measured as rate of urea production as previously described by us [11] and DDAH activity was measured as the rate of citrulline production, as optimized by us [12].

The concentration of plasma L-Arg, ADMA, and symmetric DMA was measured using reverse-phase HPLC with the Waters AccQ-Fluor fluorescent reagent kit as described by us previously [10]. Plasma citrulline was measured by a colorimetric assay [12] and $\mathrm{UNOxV}\left(\mathrm{NOx}=\mathrm{NO}_{3}+\mathrm{NO}_{2}\right)$ was measured by Griess reaction in 11 week overnight urine, as described [10]. Plasma and urine creatinine was determined by HPLC, as described previously $[3,10]$.

Plasma and renal cortical concentrations of $\mathrm{H}_{2} \mathrm{O}_{2}$ were measured using the Amplex Red Hydrogen Peroxide/Peroxidase Assay Kit (Molecular Probes, Eugene, Oreg., USA), and urinary isoprostane was detected by ELISA (Oxford Biomedical Research, Oxford, Mich., USA) as described by us [10]. 
Table 1. Effect of PAN treatment on creatinine clearance (CCr), kidney weight (KW/100 g BW) as a measure of renal hypertrophy, mean arterial BP and renal structural injury as measured by a GSI in control, LD- and HD-PAN groups

\begin{tabular}{lcll}
\hline & Control & LD-PAN & HD-PAN \\
\hline Total Ccr, ml/min/ & & & \\
$\quad 100 \mathrm{~g} \mathrm{BW}$ & $0.73 \pm 0.07$ & $0.66 \pm .09$ & $0.16 \pm 0.07^{*, * *}$ \\
$\mathrm{KW} / 100 \mathrm{~g} \mathrm{BW}$ & $0.39 \pm 0.03$ & $0.55 \pm 0.05^{*}$ & $0.79 \pm 0.07^{*, * *}$ \\
$\mathrm{BP}, \mathrm{mm} \mathrm{Hg}$ & $100 \pm 2$ & $116 \pm 5^{*}$ & $134 \pm 8^{*, * *}$ \\
GSI & $0.11 \pm 0.02$ & $1.04 \pm 0.28^{*}$ & $2.19 \pm 0.34^{*, * *}$ \\
\hline
\end{tabular}

${ }^{*} \mathrm{p}<0.05$ vs. control; ${ }^{* *} \mathrm{p}<0.05$ vs. LD-PAN.

The left kidney was fixed in 10\% formalin and paraffin embedded, and $5-\mu \mathrm{m}$ slides were stained with periodic acid Schiff (Sigma, St. Louis, Mo., USA) followed by hematoxylin. Up to one hundred glomeruli were scored, blinded, based on the $0-4+$ injury scale, and tubulointerstitial injury was graded as mild, moderate, severe or massive $[3,10]$.

\section{Statistics}

Repeated-measures ANOVA with Bonferonni post-hoc test was used for the evaluation of $\mathrm{UpV}$ and $\mathrm{BW}$ among groups. Oneway ANOVA with Newman-Keuls post-hoc test was used for evaluation of mean values across groups. Histologic data were analyzed by the Mann-Whitney $U$ test. All values are reported as means \pm SE with $\mathrm{p}<0.05$ considered statistically significant.

\section{Results}

Both LD-PAN and HD-PAN rats exhibited severe proteinuria reaching a peak at week 9 (fig. 1a). At week 11, $\mathrm{UpV}$ fell in HD-PAN associated with a very low creatinine clearance (Ccr) (table 1), while Ccr was similar to controls in LD-PAN. LD-PAN animals had similar body weight gain to control until 11 weeks (fig. 1b), whereas the body weight of HD-PAN rats remained low throughout the study. Dose-dependent increases in kidney weight, BP and glomerular damage occurred (table 1; online suppl. fig. 1; for all online suppl. material, see www. karger.com/doi/10.1159/000334740), and there was evidence of tubulointerstitial fibrosis ranging from mild to severe in the LD-PAN rats and moderate to massive in HD-PAN (online suppl. fig. 2).

At week 11, plasma citrulline increased in HD-PAN while L-Arg concentrations were unchanged (table 2). We observed dose-dependent, moderate increases in plasma ADMA in LD-and HD-PAN rats, along with a decrease

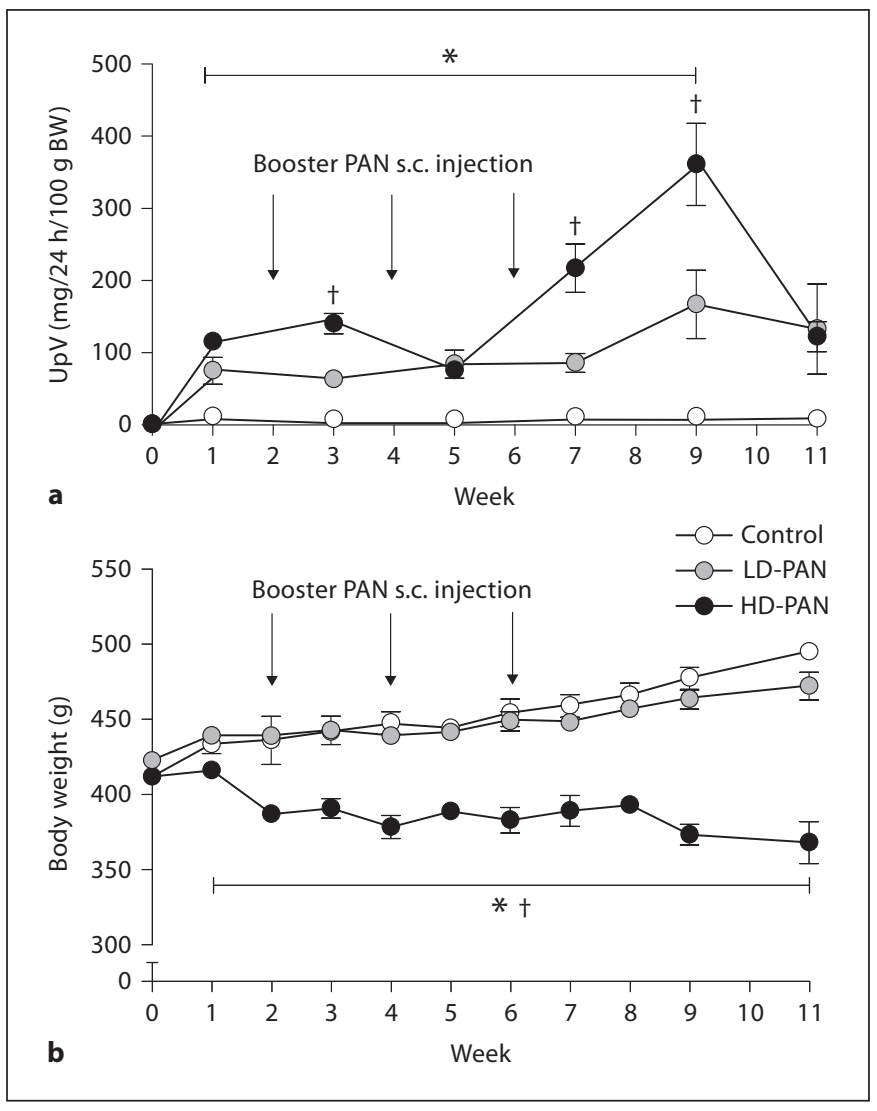

Fig. 1. Urinary protein excretion (a) and body weight (b) at baseline to 11 weeks after the initial PAN (or sham) injection. ${ }^{*} \mathrm{p}<$ 0.05 vs. control; ${ }^{\dagger} \mathrm{p}<0.05$ vs. LD-PAN.

in the L-Arg:ADMA ratio in the HD-PAN group. Plasma symmetric DMA concentration was elevated in $\mathrm{HD}$ PAN. Total NO production measured by UNOxV was similarly reduced in both PAN groups (table 2).

Kidney cortex ASS abundance fell only in HD-PAN, while ASL was reduced in both PAN groups and was undetectable with HD-PAN (fig. 2a, b). When ASS and ASL were factored for kidney weight, the same trends persisted: control and LD-PAN ASS were similar (100 \pm 8 and $125 \pm 14 \%)$ and higher than HD-PAN $(47 \pm 18 \%$; $\mathrm{p}<$ 0.01). Control ASL was higher than both LD-PAN and HD-PAN $[100 \pm 6$ vs. $43 \pm 16 \%(\mathrm{p}=0.01)$ and $1 \pm 1 \%$ $(p<0.001)]$. There was no change in hepatic ASS or ASL (fig. 2c, d).

Membrane and cytosolic expression of CAT1 was reduced in both PAN groups in kidney cortex (fig. 3a, b). In aorta, membrane-bound CAT1 was reduced in both PAN groups and increased in the cytosolic fraction with HDPAN (fig. 3c, d). 


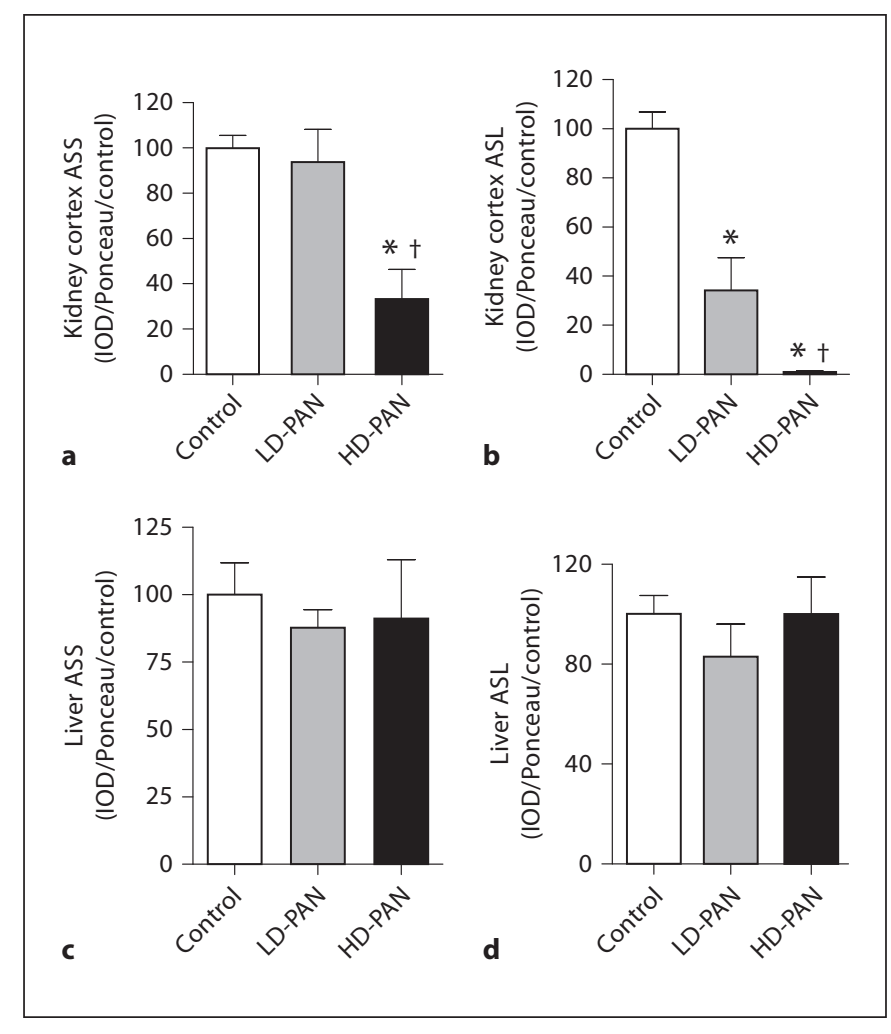

Fig. 2. ASS and ASL protein abundance in renal cortex (a) and (b) and in liver (c) and (d), respectively, measured by Western blot ( $\mathrm{n}=8$ per group). ${ }^{*} \mathrm{p}<0.05$ vs. control; ${ }^{\dagger} \mathrm{p}<0.05$ vs. LD-PAN.

Table 2. Chronic effects of $\mathrm{LD}$ and $\mathrm{HD}-\mathrm{PAN}$ on plasma citrulline (Cit), L-Arg, ADMA, L-Arg:ADMA ratio, symmetric (S)DMA, and urinary $\mathrm{NOx}$ (stable oxidation products of nitric oxide) excretion rate $\left(\mathrm{U}_{\mathrm{NOx}} \mathrm{V}\right)$ in control, LD- and $\mathrm{HD}$-PAN groups

\begin{tabular}{lccc}
\hline & Control & LD-PAN & HD-PAN \\
\hline Cit, $\mu \mathrm{mol} / \mathrm{l}$ & $89 \pm 10$ & $138 \pm 15$ & $160 \pm 23^{*}$ \\
L-Arg, $\mu \mathrm{mol} / \mathrm{l}$ & $97 \pm 9$ & $104 \pm 8$ & $98 \pm 10$ \\
ADMA, $\mu \mathrm{mol} / \mathrm{l}$ & $0.34 \pm 0.01$ & $0.50 \pm 0.02^{*}$ & $0.74 \pm 0.12^{*, * *}$ \\
L-Arg:ADMA & $283 \pm 24$ & $248 \pm 15$ & $159 \pm 30^{*, * *}$ \\
SDMA, $\mu \mathrm{mol} / \mathrm{l}$ & $0.29 \pm 0.03$ & $0.36 \pm 0.04$ & $0.92 \pm 0.18^{*, * *}$ \\
$\mathrm{U}_{\mathrm{NOx}} \mathrm{V}, \mu \mathrm{mol} / \mathrm{day} /$ & & & \\
\multicolumn{1}{c}{$100 \mathrm{~g} \mathrm{BW}$} & $0.56 \pm 0.06$ & $0.08 \pm 0.04^{*}$ & $0.09 \pm 0.05^{*}$ \\
\hline
\end{tabular}

${ }^{*} \mathrm{p}<0.05$ vs. control; ${ }^{* *} \mathrm{p}<0.05$ vs. LD-PAN.

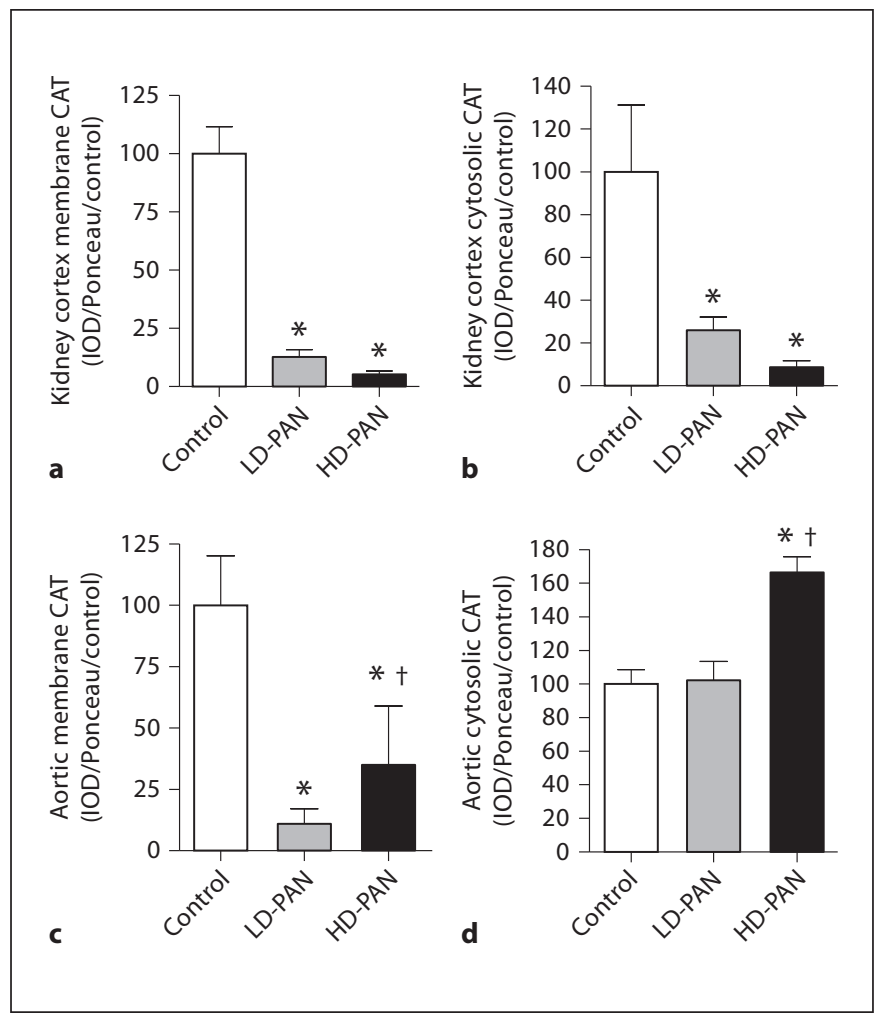

Fig. 3. Renal cortex abundance of membrane-bound (a) and cytosolic (b) CAT1 and aortic membrane-bound (c) and cytosolic CAT1 (d), measured by Western blot ( $\mathrm{n}=8$ per group). ${ }^{*} \mathrm{p}<0.05$ vs. control; ${ }^{\dagger} \mathrm{p}<0.05$ vs. LD-PAN.

Kidney cortex arginase II abundance and activity were unchanged by PAN treatment (fig. 4a, b). Neither hepatic arginase I abundance nor activity were different between groups (fig. 4c, d). Aortic expression of arginase was increased in LD-PAN but was comparable to controls in HD-PAN (fig. 4e). Arginase activity was not measured in the aorta due to lack of tissue.

To assess ADMA catabolism, we measured DDAH1 and DDAH2 protein abundance in the kidney cortex and liver. DDAH1 abundance in the kidney cortex fell with HD-PAN (fig. 5a), while an increase occurred in DDAH2 (fig. 5b). However, total kidney cortex DDAH activity fell with LD-PAN and declined further with HD-PAN (fig. 5c). There were no differences in hepatic DDAH abundance for either isoform (fig. 6a, b); however, hepatic DDAH activity decreased substantially in HD-PAN rats (fig. 6c).

To assess anti-oxidant status, we measured the abundance of the superoxide dismutase (SOD) isoforms. Although extracellular (EC) SOD kidney cortex abundance 
increased with LD-PAN, values returned to control with HD-PAN (fig. 7a). Abundance of the mitochondrial manganese $(\mathrm{Mn})$ SOD in kidney cortex decreased with both doses (fig. 7b), while the cytosolic copper-zinc (CuZn) SOD decreased with HD-PAN (fig. 7c). We also measured indices of oxidative stress in the kidney cortex (as a marker of local production) as well as in the plasma and urine (for global indices of oxidative stress). We did not see changes in $\mathrm{H}_{2} \mathrm{O}_{2}$ levels in kidney cortex or plasma; however, the urinary excretion rate of $\mathrm{H}_{2} \mathrm{O}_{2}$ was increased in the LD-PAN group (table 3). In contrast, kidney cortex p22phox abundance, the subunit of NADPH oxidase that is critical for superoxide generation in the kidney, and urinary excretion of the lipid oxidation marker, F2-isoprostanes $\left(\mathrm{U}_{\mathrm{F} 2 \text {-iso }} \mathrm{V}\right)$, increased significantly with $\mathrm{HD}$ PAN compared to both control and LD-PAN (table 3).

\section{Discussion}

The novel findings of this study are that PAN-induced $\mathrm{CKD}$ results in a severity-dependent reduction in renal ASS/ASL abundance. This implies reduced renal L-Arg synthesis which, together with loss of renal cortical CAT1, suggests reduced renal L-Arg release. We also find loss of functional vascular (aortic) CAT1, which will inhibit LArg uptake from plasma and may account for the 'deceptively' normal plasma L-Arg. We see no evidence that activation of renal arginase contributes to decreased L-Arg availability, although the increased vascular (aortic) arginase during development of mild hypertension in LDPAN may contribute to vascular NO deficiency. Plasma ADMA increased moderately in a dose-dependent manner with PAN-induced CKD and varied inversely with renal DDAH activity. A fall in hepatic DDAH activity, reduced renal clearance and increased oxidative stress markers (renal cortex p22 phox abundance and F2-isoprostane excretion) in the severe injury model had no further impact on plasma ADMA. PAN initially produces nephrotic syndrome, which, when followed by additional, lower doses, converts to progressive CKD [13]. In the present study, we found that PAN resulted in dosedependent glomerular sclerosis and tubulointerstitial fibrosis such that after 11 weeks of HD-PAN the kidneys were severely injured with only $\sim 20 \%$ residual renal function. A significant but moderate renal injury without functional impairment was seen with LD-PAN. A dosedependent mild-to-moderate hypertension was also seen with PAN. As CKD progresses, increases in plasma citrulline are invariably observed once GFR falls [14], and

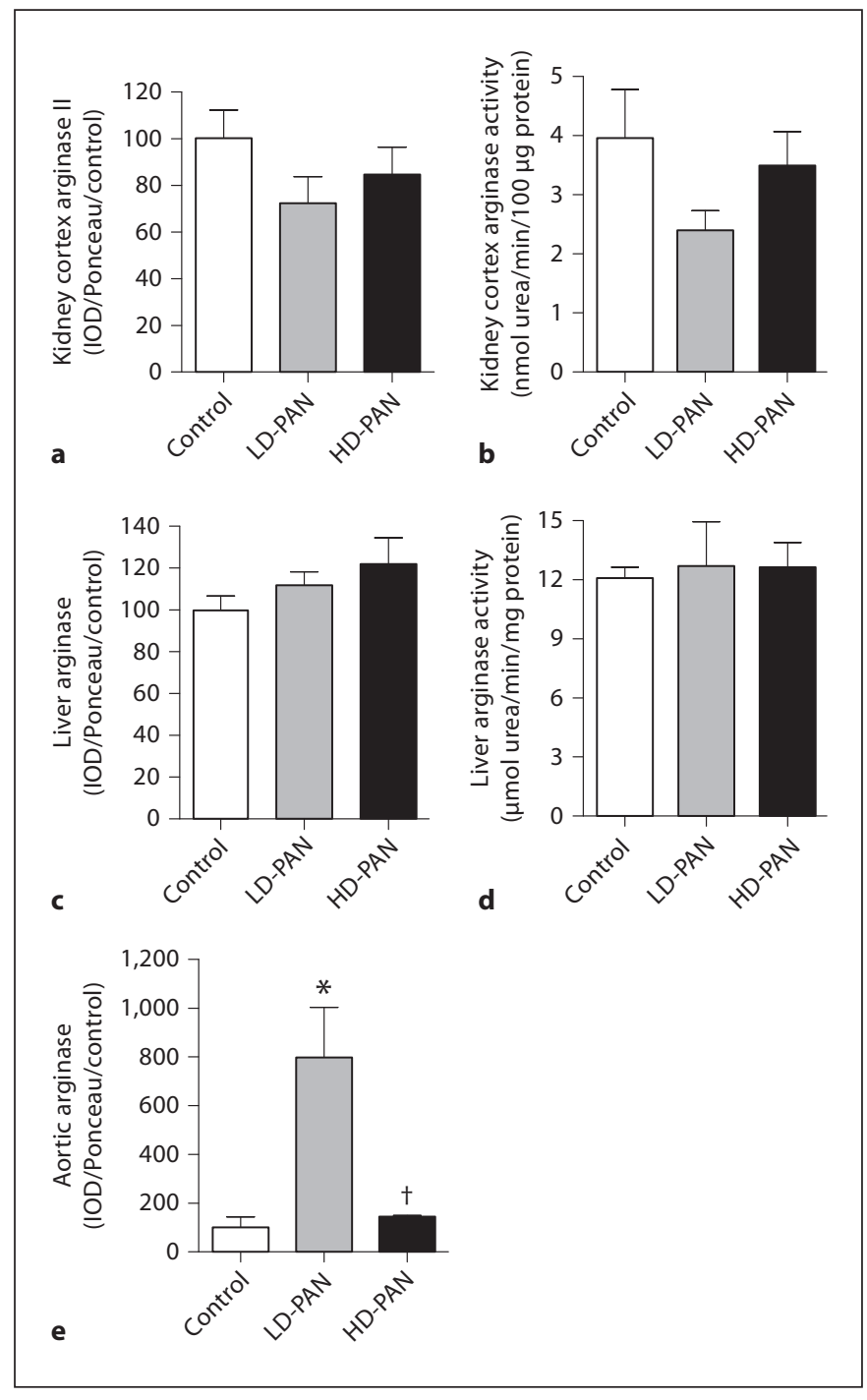

Fig. 4. Kidney cortex arginase II protein abundance (a) measured by Western blot and arginase activity (b) measured by urea formation ( $\mathrm{n}=8$ per group). Hepatic arginase I protein (c) and activity (d). Aortic arginase I protein (e). ${ }^{*} \mathrm{p}<0.05$ vs. control; ${ }^{\dagger} \mathrm{p}<0.05$ vs. LD-PAN.

this was seen with HD-PAN in the present study. Citrulline is rate-limiting for L-Arg production [2], and the increases in plasma citrulline suggest decreased renal uptake/utilization, presumably due to reductions in renal ASS/ASL abundance leading to reduced renal L-Arg synthesis. In fact, renal ASL was undetectable in HD-PAN, which would prevent any renal L-Arg synthesis. We previously reported a reduction in renal ASS in CKD due to RMR where we also observed marked falls in renal release of L-Arg [3]. Any L-Arg produced by the kidney 
Fig. 5. Effect of PAN treatment on DDAH isoforms 1 (a) and 2 (b) abundance, and total DDAH activity (c) in the kidney cortex of the control, LD- and HD-PAN groups. ${ }^{*} \mathrm{p}<0.05$ vs. control; ${ }^{\dagger} \mathrm{p}<0.05$ vs. LD-PAN.

Fig. 6. Effect of PAN treatment on DDAH isoforms 1 (a) and 2 (b) abundance, and total DDAH activity (c) in the liver of the control, LD- and HD-PAN groups. ${ }^{*} \mathrm{p}<$ 0.05 vs. control; ${ }^{\dagger} \mathrm{p}<0.05$ vs. LD-PAN.
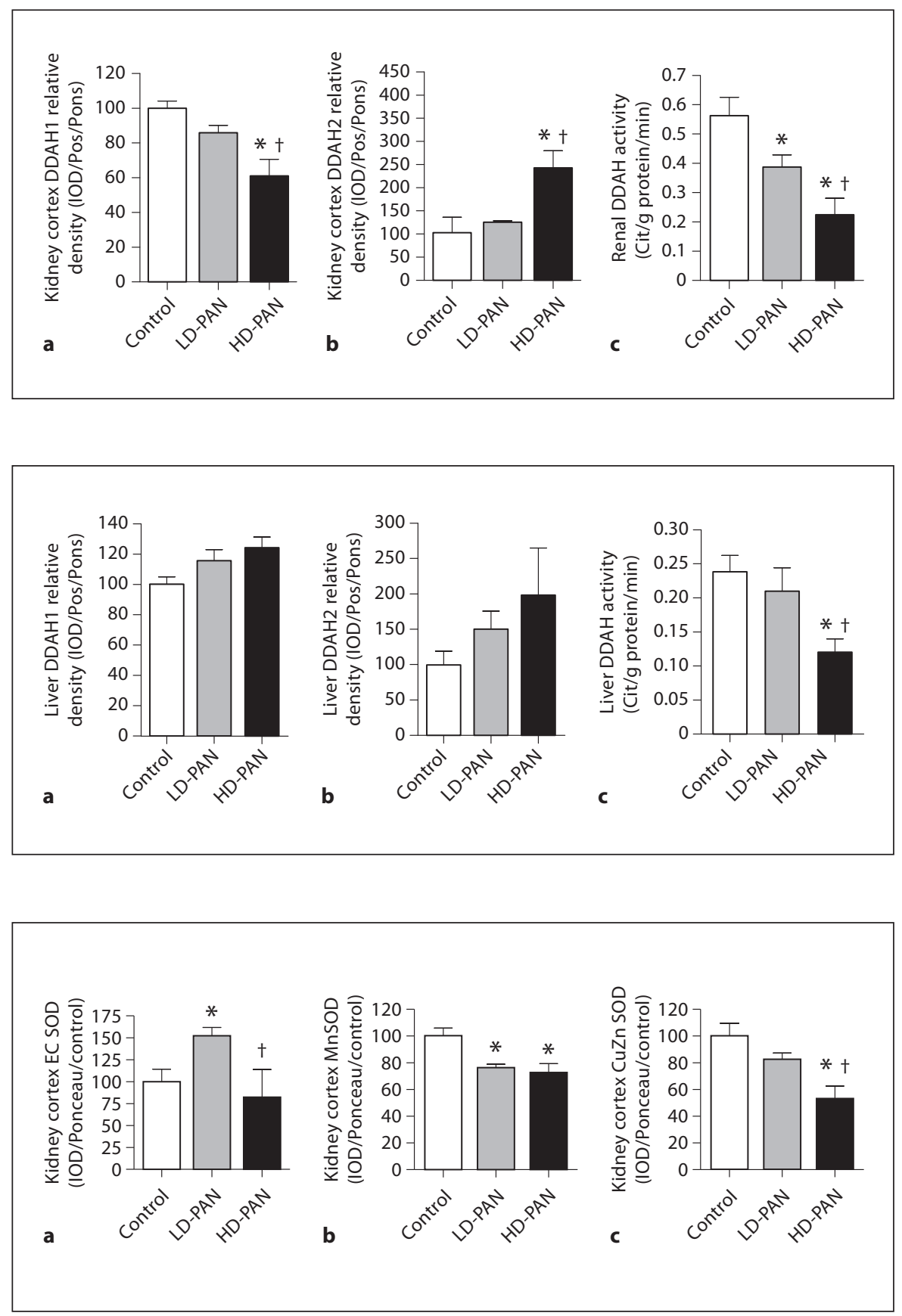

Fig. 7. Effect of PAN treatment on extracellular superoxide dismutase (EC SOD; a), mitochondrial SOD (Mn SOD; b), and cytosolic SOD (CuZn SOD; c) abundance in the kidney cortex of the control, LDand HD-PAN groups. ${ }^{*} \mathrm{p}<0.05$ vs. control; ${ }^{\dagger} \mathrm{p}<0.05$ vs. LD-PAN. must be secreted across the proximal peritubular membrane in order to enter the plasma and this requires membrane transport. CAT1 is present on the peritubular side of renal epithelial cells [4], and we observed a reduction in renal cortical membrane CAT1 abundance. Since the majority of cortical tissue is proximal tubule, this suggests a decreased efflux capacity from the site of synthesis. Taken together, falls in renal L-Arg synthesis and re- nal L-Arg efflux capacity should lead to falls in plasma L-Arg.

This brings us to the puzzling finding that plasma LArg levels are usually normal in CKD and ESKD [1] and are maintained with RMR [3] and in the present study. The normal kidney contributes the majority of circulating L-Arg [1,2], and with the loss of renal ASS/ASL in both PAN groups the maintenance of plasma levels is unex- 
Table 3. Effect of PAN on oxidative stress as measured by hydrogen peroxide levels in the kidney cortex, plasma $\left(\mathrm{P}_{\mathrm{H}_{2} \mathrm{O}_{2}}\right)$ and urine excretion rate $\left(\mathrm{U}_{\mathrm{H}_{2} \mathrm{O}_{2}} \mathrm{~V}\right)$, kidney cortex abundance of the NADPH oxidase subunit, p22phox, and urinary excretion rate of $\mathrm{F} 2$-isoprostanes $\left(\mathrm{U}_{\mathrm{F}_{2}-\mathrm{Iso}} \mathrm{V}\right)$

\begin{tabular}{lccc}
\hline & Control & LD-PAN & HD-PAN \\
\hline Hydrogen peroxide & & & \\
Kidney cortex, nmol/mg & $0.07 \pm 0.01$ & $0.11 \pm 0.03$ & $0.30 \pm 0.22$ \\
$\mathrm{P}_{\mathrm{H}_{2} \mathrm{O}_{2}, \mu \mathrm{mol} / \mathrm{l}}$ & $2.0 \pm 0.5$ & $9.6 \pm 3.2$ & $7.3 \pm 0.9$ \\
$\mathrm{U}_{\mathrm{H}_{2} \mathrm{O}_{2} \mathrm{~V}, \mathrm{nmol} / 24 \mathrm{~h} / 100 \mathrm{~g} \mathrm{BW}}$ & $9 \pm 1$ & $19 \pm 4^{*}$ & $12 \pm 2$ \\
Kidney cortex p22phox, IOD/Ponceau/control & $100 \pm 13$ & $108 \pm 13$ & $266 \pm 21^{* * * *}$ \\
$\mathrm{U}_{\mathrm{F}_{2} \text {-iso } \mathrm{V}, \mathrm{nmol} / 24 \mathrm{~h} / 100 \mathrm{~g} \mathrm{BW}}$ & $3.2 \pm 0.4$ & $4.8 \pm 0.8$ & $8.5 \pm 1.0^{* * * *}$ \\
\hline${ }^{*} \mathrm{p}<0.05$ vs. control; ${ }^{* *} \mathrm{p}<0.05$ vs. LD-PAN. & & & \\
\hline
\end{tabular}

pected. One explanation is that a reduction in endothelial L-Arg uptake occurs due to the fall in vascular endothelial L-Arg uptake. CATs are involved in the influx as well as the efflux of cationic amino acids, and CAT1 is the primary transporter for L-Arg into the vascular endothelium [5]. We saw a marked fall in the membrane-bound CAT1 from aorta of both PAN models, and we previously reported that protein abundance of aortic and glomerular CAT1 was reduced in RMR-induced CKD [6]. Thus, reduced endothelial L-Arg uptake may camouflage deficient renal L-Arg production by allowing accumulation to apparently normal plasma values. Loss of endothelial L-Arg uptake could also explain the failure of L-Arg supplementation to improve endothelial function or reduce the rate of progression in adults or children with CKD [1, $15,16]$.

In addition to decreased renal synthesis, L-Arg deficiency in CKD could occur due to increased metabolism by arginase, which is activated by injury and is involved in repair [17]. Increased vascular arginase has been reported in several models of hypertension and CKD [1]. In the present study, we did see increased aortic arginase abundance with LD-PAN, but with HD-PAN aortic arginase was comparable to control levels, perhaps reflecting more rapid progression of the injury and higher $\mathrm{BP}$ in these animals such that an attempt at repair had already occurred. There were no changes in renal or hepatic arginase abundance/activity in either PAN group. Overall, there is no indication that renal or hepatic arginase activation consumes excess L-Arg during progression of PAN-CKD, although it is possible that vascular arginase activation in the LD-PAN group will impair endothelial NO production as the initial mild hypertension develops.
We also observed moderate increases in plasma ADMA in PAN-induced CKD, confirming the findings of Sato et al. [9]. Plasma ADMA is closely linked to cardiovascular risk, even in 'normal' individuals, and also in ESKD where values are high and variable and predict cardiovascular and all-cause mortality [8]. In CKD, plasma ADMA increases in some patients but is variable [18], which suggests that loss of renal clearance is not the only factor determining plasma ADMA in renal disease [1]. It is currently believed that plasma ADMA is regulated primarily by DDAH1 activity in kidney and liver [7]. Here, we observed a small rise in plasma ADMA in LD-PAN in association with reduced renal DDAH activity. With a further loss of renal DDAH activity, there was a small further increase in plasma ADMA. However, falls in renal clearance and hepatic DDAH activity as well as increased oxidative stress did not produce additive effects to increase plasma ADMA in HD-PAN. Thus, plasma ADMA is not simply the end result of kidney clearance and kidney and liver DDAH activities but likely also reflects tissue production and efflux rates as well as DDAH catabolic activity in other locations. Indeed, in DDAH1 knockout mice, endothelial specific DDAH1 knockout produces increased plasma ADMA and hypertension and has the same phenotype as a global DDAH1 knockout model $[19,20]$, suggesting that vascular DDAH1 activity can play a major role in regulating plasma ADMA. We also observed that with high-dose PAN, the fall in hepatic DDAH activity occurred in the absence of any change in DDAH1 or DDAH2 abundance. This finding supports the concept that post-translational modifications occur to the DDAH enzymes [7] and also highlights the importance of conducting measures of enzyme activity in addition to abundance for these proteins. 
Extensive post-translational modification of DDAH activity occurs due to both nitrosylation and oxidation which reduce activity [7]. Here, we see decreased MnSOD and CuZnSOD abundance with HD-PAN, suggesting decreased renal antioxidant capacity. In addition, the abundance of p22phox, which is critical to the activity of the NADPH oxidase complex [21], was increased with HDPAN, and urinary isoprostane excretion increased with HD-PAN, suggesting widespread lipid peroxidation. We also observed increased $\mathrm{H}_{2} \mathrm{O}_{2}$ excretion in LD-PAN. Other investigators report increased oxidative stress in the PAN model of CKD using various indices [22, 23]. Overall, we do see evidence of increased oxidative stress, particularly in the HD-PAN rats, and this may explain the reduced hepatic DDAH activity in the absence of a fall in DDAH protein abundance.

In conclusion, in the PAN-induced model of CKD, we see marked declines in total NO production. Decreased renal L-Arg synthesis/release decreased vascular L-Arg transporters (limiting endothelial L-Arg uptake) and increased plasma ADMA could all contribute. Future studies are indicated to directly measure renal L-Arg production as well as total body L-Arg production and turnover in PAN-induced CKD. These could include determination of L-Arg efflux from kidney and influx into vasculature, to assess the importance of loss of renal and vascular CAT1. The increased plasma ADMA was associated with loss of renal DDAH activity in LD-PAN rats, and in HDPAN a further decline in renal DDAH activity was associated with a further small increase in plasma ADMA. However, with HD-PAN there was also loss of renal clearance, falls in hepatic DDAH activity and evidence of widespread oxidative stress; all effects predicted to exacerbate a rise in plasma $\mathrm{ADMA}$. This suggests that control of plasma ADMA may be more complex than currently believed. We need to expand our understanding of the relative importance of the various organs and the two isoforms of DDAH in ADMA clearance, since these are potential novel therapeutic targets for treatment of CKD and cardiovascular disease. Also, a more precise understanding of the type, time course and location of oxidative stress that arises during CKD is urgently needed, so that specific targeted antioxidant interventions can be employed.

\section{Acknowledgements}

These studies were funded by NIH grant R01DK56843 (C.B.) and stipend support from T32DK076541 (N.C.M.) and T32HL083810 (J.M.S.)

\section{Disclosure Statement}

None of the authors have any conflicts of interest to disclose.

\section{References}

1 Baylis C: Arginine, arginine analogs and nitric oxide production in chronic kidney disease. Nat Clin Pract Nephrol 2006:2:209-220.

-2 Wu G, Morris SM Jr: Arginine metabolism: nitric oxide and beyond. Biochem J 1998:336: $1-17$.

$\checkmark 3$ Chen GF, Baylis C: In vivo renal arginine release is impaired throughout development of chronic kidney disease. Am J Physiol Renal Physiol 2010:298:F95-F102.

4 Kizhatil K, Albritton LM: System y+ localizes to different membrane subdomains in the basolateral plasma membrane of epithelial cells. Am J Physiol Cell Physiol 2002: 283:C1784-1794.

5 Closs EI, Boissel JP, Habermeier A, Rotmann A: Structure and function of cationic amino acid transporters (CATs). J Membr Biol 2006: 213:67-77.

6 Schwartz IF, Ayalon R, Chernichovski T, Reshef R, Chernin G, Weinstein T, Litvak A, Levo Y, Schwartz D: Arginine uptake is attenuated through modulation of cationic amino-acid transporter-1, in uremic rats. Kidney Int 2006:69:298-303.
7 Palm F, Onozato ML, Luo Z, Wilcox CS: Dimethylarginine dimethylaminohydrolase (DDAH): expression, regulation, and function in the cardiovascular and renal systems. Am J Physiol Heart Circ Physiol 2007; 293:H3227-H3245.

$>$ Böger RH, Maas R, Schulze F, Schwedhelm E: Asymmetric dimethylarginine (ADMA) as a prospective marker of cardiovascular disease and mortality: an update on patient populations with a wide range of cardiovascular risk. Pharmacol Res 2009;60:481-487.

$\checkmark 9$ Sato J, Masuda H, Tamaoki S, Hamasaki H, Ishizaka K, Matsubara O, Azuma H: Endogenous asymmetrical dimethylarginine and hypertension associated with puromycin nephrosis in the rat. Br J Pharmacol 1998;125: 469-476.

10 Sasser JM, Moningka NC, Cunningham MW Jr, Croker BP, Baylis C: Asymmetric dimethylarginine in angiotensin II induced hypertension. Am J Physiol Regul Integr Comp Physiol 2009;298:R740-R746.
11 Zharikov S, Krotova K, Hu H, Baylis C, Johnson RJ, Block ER, Patel J: Uric acid decreases NO production and increases arginase activity in cultured pulmonary artery endothelial cells. Am J Physiol Cell Physiol 2008: 295:C1183-C1190

12 Tain YL, Baylis C: Determination of dimethylarginine dimethylaminohydrolase activity in the kidney. Kidney Int 2007:72:886-889.

13 Erdely A, Freshour G, Smith C, Engels K, Olson JL, Baylis C: Protection against puromycin aminonucleoside-induced chronic renal disease in the Wistar-Furth rat. Am J Physiol Renal Physiol 2004:287:81-89.

14 Chan W, Wang M, Kopple JD, Swendseid ME: Citrulline levels and urea cycle enzymes in uremic rats. J Nutr 1974:104:678-683.

15 Cross JM, Donald AE, Kharbanda R, Deanfield JE, Woolfson RG, MacAllister RJ: Acute administration of L-arginine does not improve arterial endothelial function in chronic renal failure. Kidney Int 2001;60:23182323 
-16 Bennett-Richards KJ, Kattenhorn M, Donald AE, Oakley GR, Varghese Z, Bruckdorfer KR, Deanfield JE, Rees L: Oral L-arginine does not improve endothelial dysfunction in children with chronic renal failure. Kidney Int 2002:62:1372-1378.

-17 Wei LH, Wu G, Morris SM, Ignarro LJ: Elevated arginase I expression in rat aortic smooth muscle cells increases cell proliferation. Proc Natl Acad Sci USA 2001:98:92609264.

18 Jacobi J, Tsao PS: Asymmetrical dimethylarginine in renal disease: limits of variation or variation limits? A systematic review. Am J Nephrol 2008;28:224-237.
$19 \mathrm{Hu}$ X, Xu X, Zhu G, et al: Vascular endothelial-specific dimethylarginine dimethylaminohydrolase-1-deficient mice reveal that vascular endothelium plays an important role in removing asymmetric dimethylarginine. Circulation 2009; 120:2222-2229.

$20 \mathrm{Hu}$ X, Atzler D, Xu X, et al: Dimethylarginine dimethylaminohydrolase- 1 is the critical enzyme for degrading the cardiovascular risk factor asymmetrical dimethylarginine. Arterioscler Thromb Vasc Biol 2011;31: 1540-1546.
21 Modlinger P, Chabrashvili T, Gill PS, Mendonca M, Harrison DG, Griendling KK, Li M, Raggio J, Wellstein A, Chen Y, Welch WJ, Wilcox CS: RNA silencing in vivo reveals role of $\mathrm{p} 22^{\text {phox }}$ in rat angiotensin slow pressor response. Hypertension 2006;47:238-244.

22 Rincon J, Romero M, Viera N, Pedreanez A, Mosquera J: Increased oxidative stress and apoptosis in acute puromycin aminonucleoside nephrosis. Int J Exp Pathol 2004;85:2533.

23 Marshall CB, Pippin JW, Krofft RD, Shankland SJ: Puromycin aminonucleoside induces oxidant-dependent DNA damage in podocytes in vitro and in vivo. Kidney Int 2006; 70:1962-1973. 